\section{Histoire Épistémologie Langage}

42-2 | 2020

Genèse, origine, récapitulation. Trân Đức Thảo face aux sciences du langage

\title{
Trần Đức Thảo en contexte. Une critique originale
}

de Saussure?

Juliette Farjat

\section{CpenEdition}

\section{Journals}

Édition électronique

URL : https://journals.openedition.org/hel/376

DOI : $10.4000 /$ hel.376

ISSN : 1638-1580

Éditeur

Société d'histoire et d'épistémologie des sciences du langage

Édition imprimée

Date de publication : 31 décembre 2020

Pagination : $77-91$

ISBN : 979-10-91587-13-6

ISSN : 0750-8069

Référence électronique

Juliette Farjat, «Trần Đức Thảo en contexte. Une critique originale de Saussure ? », Histoire

Épistémologie Langage [En ligne], 42-2 | 2020, mis en ligne le 28 octobre 2021, consulté le 29 octobre 2021. URL : http://journals.openedition.org/hel/376 ; DOI : https://doi.org/10.4000/hel.376

Ce document a été généré automatiquement le 29 octobre 2021.

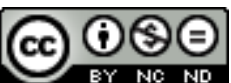

HEL is licensed under a Creative Commons Attribution-NonCommercial-NoDerivatives 4.0 International License 


\title{
Trần Đức Thảo en contexte. Une critique originale de Saussure?
}

\author{
Juliette Farjat
}

Je tiens à remercier chaleureusement les relecteurs et les responsables de la revue HEL, ainsi que Jacopo D'Alonzo, pour leur travail de relecture et pour leurs commentaires à propos de cet article.

\section{Introduction}

1 Les deux principales objections que Trần Đức Thảo adresse à la linguistique saussurienne ont été, l'une et l'autre, développées au sein de deux espaces de discussions théoriques qui appartiennent à la tradition marxiste ${ }^{1}$. La première s'adresse à l'abstraction de la théorie linguistique de Saussure : elle s'élabore parmi les linguistes russes dans les années 1920-1930, en particulier chez Valentin Vološinov et Lev Jakubinskij (Tylkowski 2012, Jakubinskij 2012). La seconde consiste en la remise en cause de la conception saussurienne de la valeur des signes à partir du modèle de la critique de l'économie politique, telle qu'elle est élaborée par Marx : elle se développe dans les années 1960-1970, dans les sciences sociales et en philosophie, notamment chez Henri Lefebvre, Jean-Joseph Goux, ou Ferruccio Rossi-Landi.

2 On tentera d'abord, dans une perspective comparative, de rendre compte de la manière dont Thảo développe ses deux objections principales à l'égard de Saussure. Nous considérerons ces critiques pour leur intérêt propre, sans juger de leur légitimité et sans examiner la validité des positions qui sont attribuées à Saussure : elles seraient sans doute, pour la plupart, largement contestées aujourd'hui. On cherchera ensuite à comprendre le lien qui unit ces deux critiques et à mettre en avant les indications positives qu'elles fournissent en faveur d'une conception marxiste de la nature et de la place du langage dans la société.

Précisons tout de suite que la plupart des reproches qui sont faits à l'égard de la théorie saussurienne relèvent d'une critique externe plutôt que d'une critique interne. En effet, Thảo et les autres auteurs dont il sera question ici reprochent moins à Saussure la 
manière dont il traite l'objet qu'il s'est donné - la langue comme système de signes que le choix même de cet objet. Si Saussure affirme explicitement que la langue est un objet qui, pour les besoins de la science, a été sélectionné et isolé à l'intérieur de ce tout «multiforme et hétéroclite» qu'est le langage (Saussure 1987 [1916]: 25), c'est précisément cet isolement qui lui est reproché, dans la mesure où il empêcherait la prise en compte d'un certain nombre de phénomènes qui, du point de vue de Thảo, sont essentiels pour comprendre la spécificité du langage humain.

\section{L'abstraction de la linguistique saussurienne et l'arbitraire du signe}

4 En Russie, le processus révolutionnaire et les problèmes de politique langagière qui l'accompagnent engagent des discussions théoriques importantes dans le champ de la linguistique. La réception de Saussure est alors divisée entre l'école de Moscou dite "formaliste», majoritairement favorable à la théorie saussurienne, et l'école de Leningrad qui s'y oppose (Ageeva 2009). C'est à cette dernière école qu'appartiennent Valentin Vološinov, auteur désormais reconnu de l'ouvrage Marxisme et philosophie du langage (Vološinov 2010), et Lev Jakubinskij, souvent considéré comme étant à l'origine de la sociolinguistique en Russie. Ces deux linguistes ont en commun avec Thảo de proposer une critique de l'abstraction de la linguistique de Saussure.

5 Dans Marxisme et philosophie du langage, Valentin Vološinov engage une critique de l'«objectivisme abstrait» de la théorie saussurienne. Ses diverses objections se ramènent au refus de la définition saussurienne de la langue comme objet propre de la linguistique. En la réduisant à l'étude d'un système formel, qui exclut la prise en compte de l'activité langagière réelle qu'est la parole, ainsi que l'analyse de ses évolutions historiques, Saussure érigerait en objet de science une abstraction qui ne correspondrait à aucun point de vue légitime. En effet, la langue comme système de signes n'existe pas objectivement : la langue n'est à aucun moment figée dans un tel système, au contraire, elle ne cesse d'évoluer à chaque instant. Et elle n'existe pas non plus subjectivement: les sujets parlants ne considèrent jamais les paroles qu'ils entendent ou prononcent comme des éléments d'un système déjà constitué. Ils sont au contraire tout entiers tournés vers la nouveauté de ce qu'il y a à dire ou à entendre et qui dépend autant du contexte d'énonciation que de la langue elle-même ${ }^{2}$ : les paroles sont moins des signes organisés selon des règles que des vérités et des mensonges, des ordres et des conseils, etc. Vološinov propose alors d'analyser la langue dans son historicité et ses variations, qui, loin d'être réductibles à des accidents, en constituent l'essence, et de considérer les mots non comme des signes tirant leur signification de leurs rapports réciproques, mais comme étant chargés d'une valeur idéologique dépendante d'un contexte d'interaction linguistique effectif dont on ne peut les séparer.

6 Si Lev Jakubinskij partage le diagnostic porté par Vološinov, il cherche également à en analyser la portée générale en l'interprétant comme la marque d'une approche idéologique en linguistique, c'est-à-dire d'une approche qui contribue à la préservation de l'ordre existant. Dans le contexte qui est le sien, où se pose vivement la question de savoir si une politique linguistique peut être mise en place en URSS, Jakubinskij reproche à Saussure de définir la langue de manière à refuser aux sujets toute emprise sur cette dernière et donc toute possibilité d'en entreprendre la réforme (Jakubinskij 
2012 : 181-212). Pour Saussure (1987 [1916] : 107-108), en effet, « La langue est de toutes les institutions sociales celle qui offre le moins de prise aux initiatives ». Ainsi, la préexistence d'une langue sur les individus qui la parlent, le caractère arbitraire du signe et l'impossibilité de s'extraire de la langue pour pouvoir la réformer sont autant d'arguments qui justifient, selon Jakubinskij (2012: 181), «l'impossibilité d'une politique linguistique». Notons que ce type de critique a eu des échos plus récents. Selon Marcellesi et Gardin (1973: 186), le caractère idéologique d'une telle thèse apparaîtrait dans le glissement qui ferait passer Saussure, dans le chapitre sur la "Propagation des ondes linguistiques», du registre descriptif au registre normatif. Pour eux, Saussure, tout en prétendant décrire un état de fait - la tendance de la langue à l'inertie -, ne pourrait s'empêcher d'accorder une valeur positive à cette inertie et d'associer une valeur négative au changement, en le décrivant comme le résultat d'une action dissolvante de "l'esprit de clocher» et en le qualifiant de "trouble", d'obscurcissement ou de complication du mécanisme linguistique (Saussure 1987 [1916] : 281-282). Dans ces passages, il chercherait donc à faire passer sa prise de parti en faveur de la conservation de la langue pour une simple description scientifique et à absolutiser le système linguistique actuel.

7 Dans ses deux articles de La Nouvelle Critique, Thảo parcourt le même chemin que les deux linguistes russes. Mais chez lui, le caractère abstrait de l'objet «langue » est critiqué à partir de la thèse centrale de l'arbitraire du signe qui n'occupait chez eux qu'une place secondaire. Il identifie ainsi le noyau de la doctrine saussurienne dont la critique remettrait en question l'ensemble du système. Thảo attribue à Saussure deux définitions de l'arbitraire du signe linguistique. Le signe est d'abord arbitraire en ce que rien dans le "concept signifié » ne contraint ou ne détermine le signifiant. Ainsi, la sélection de tel signifiant correspondant à tel signifié est immotivée. Il est arbitraire ensuite en ce que les catégories de la langue ne sont pas déterminées par des catégories réelles ou par une division objective de la réalité extérieure (Saussure 1987 [1916] : 97, 58).

8 Thảo rejette l'absolutisation de ce principe de l'arbitraire du signe dans les deux sens qui lui sont accordés. Premièrement, il associe la conception arbitraire des signes à une double réduction. D'une part, les signes en général se trouvent réduits aux signes linguistiques, si bien que Saussure, tout en reconnaissant l'existence de signes naturels comme les signes imitatifs ou les symboles, finit par faire du signe linguistique arbitraire un idéal sémiologique et par considérer les autres signes en défaut par rapport à cet idéal. D'autre part, l'expression linguistique est comprise relativement au modèle réducteur d'un langage scientifique dont la réalisation la plus aboutie serait le langage purement conventionnel de l'algèbre.

9 S'il s'agit bien pour Saussure d'un modèle et non d'une prétention à dire ce qu'est effectivement la langue, Thảo reproche à Saussure le choix de ce modèle, qui s'avère selon lui incapable de rendre compte de ce qui lui paraît essentiel, à savoir la parole ordinaire. D'après Thảo, celle-ci est en effet orientée par un idéal inverse : celui d'atteindre directement et sans médiation celui à qui l'on s'adresse. À l'image de l'expression artistique, les intonations, les mimiques, les gestes qui accompagnent la parole contribueraient à la production d'un sens indépendamment de la neutralité des conventions, si bien qu'il serait tout autant possible de fonder la sémiologie sur un modèle « esthétique » (ou expressif) que sur un modèle conventionnel. Ce ne serait que dans un second temps, à partir d'un processus d'abstraction, que les signes en 
viendraient à revêtir un caractère arbitraire. La preuve en est, d'après Thảo, qu'un étranger tente toujours de s'exprimer à l'aide de gestes imitatifs, directement compréhensibles et qui représenteraient, mieux que le langage articulé, la nature non entièrement arbitraire de la communication. Le philosophe vietnamien oppose ainsi "le système général des signes intrinsèques, ou esthétiques [...] caractérisés par l'expressivité intrinsèque du signifiant » et "le système général des signes arbitraires, posés par la convention sociale». Bien évidemment, conclut-il «c'est le premier système qui fonde le second, puisqu'il présente directement dans l'intuition sensible le contenu de signification, auquel le second donne une expression conventionnelle " (Thảo $1974: 40$ ).

Deuxièmement, l'hypothèse de l'arbitraire du signe au sens d'une indépendance des signifiés à l'égard du réel constituerait, pour lui, un postulat idéaliste : parce qu'il tire sa signification de son rapport aux autres signes plutôt que de la réalité, «le concept, présenté ainsi à la manière hégélienne comme pure forme de négation, n'a plus alors d'autre contenu de connaissance que la simple structure linguistique de son propre langage, de sorte que le savoir revient comme chez Hegel à un pur savoir de soi » (ibid.). Cette critique de l'«idéalisme" saussurien est une constante au sein des critiques marxistes soviétiques de Saussure. C'est en particulier celle qu'identifie Patrick Sériot (2020) : elle revient à reprocher à Saussure d'ignorer le rapport entre les signes d'un côté et la réalité de l'autre, et de permettre ainsi de «douter de l'existence des objets matériels». Comme le montre Sériot, cette critique repose sur une interprétation ontologique de l'épistémologie saussurienne. Or « Saussure [...] n'a jamais envisagé que la réalité "n'existe pas" (position ontologique), il se place sur un autre terrain, qui distingue le signifié du référent, lequel n'est pas pertinent pour la théorie ». Il en résulte cependant pour Thảo que les prétentions scientifiques de la linguistique saussurienne s'évanouiraient d'elles-mêmes. Il remarque ainsi que Saussure en vient inévitablement à se contredire lorsqu'il affirme par exemple qu'il ne faut pas se fier aux mots et à leur usage pour distinguer langue, langage et parole, mais bien aux choses qu'on cherche à définir. Après avoir distingué ces trois concepts, Saussure (1987 [1916] : 31) écrit en effet: «Il est à remarquer que nous avons défini des choses et non des mots; les distinctions établies n'ont donc rien à redouter de certains termes ambigus qui ne se recouvrent pas d'une langue à l'autre. [...] C'est une mauvaise méthode que de partir des mots pour définir les choses. » Par là, Saussure admettrait que les mots doivent bien avoir un lien avec le contenu objectif du réel qu'ils peuvent représenter de manière plus ou moins confuse. Pour Thảo (1974 : 41) :

[...] en montrant que le langage de la science vise à définir à travers ses concepts non pas des mots mais bien les choses elles-mêmes, l'auteur [Saussure] montrait un solide bon sens scientifique [...]. Or, il est clair que pour « définir les choses », il faut bien employer, en dernière analyse, les mots du langage ordinaire. [...] Et puisque la définition scientifique, comme Saussure l'affirme à juste titre, porte sur la réalité même des choses, il faut bien admettre que le langage ordinaire implique déjà dans ses significations verbales spontanément constituées un contenu de connaissance authentique, reproduisant de manière véridique, bien que partielle et plus ou moins confuse, le contenu objectif du réel, de sorte que ce contenu donné dans la signification des mots peut être précisé et développé par les combinaisons du discours, mais ne saurait en aucune façon se ramener au simple rapport des mots entre eux. 
11 Il y aurait donc une contradiction entre le constructivisme théorique de Saussure et son réalisme pratique que Thảo $(1974: 41)$ qualifie de «matérialisme spontané des savants » (l'expression vient de Lénine 1948 [1909]).

12 Notons que le principe de l'arbitraire du signe est qualifié par Thảo d'« idéalisme ", et d'«objectivisme abstrait» par Vološinov (par différence avec «le subjectivisme idéaliste » d'un Croce ou d'un Vossler). En réalité, Vološinov accuse d'idéalisme toute théorie qui accorde un primat à la pensée individuelle sur la langue comme si la première était créatrice de la seconde: l'idéalisme est donc pour lui une certaine manière d'envisager les rapports entre pensée (spirituelle et subjective) et langue (matérielle et sociale). Pour Thảo, en revanche, la spécificité de l'approche idéaliste (qu'il attribue à Saussure) est qu'elle accorde une autonomie telle à la langue qu'elle serait sans lien avec la réalité objective : l'idéalisme est alors une manière d'envisager les rapports, non plus entre la pensée et le langage, mais entre le langage et la réalité matérielle. Ainsi, si la thèse de l'arbitraire du signe est objectiviste pour Vološinov au sens où elle fige les signes linguistiques en conventions neutres indépendantes des sujets et du contexte, elle est idéaliste pour Thảo au sens où elle sépare les signes du monde concret que pourtant, ils visent ${ }^{3}$. C'est la réalité sociale des sujets et du contexte d'énonciation que Vološinov met en avant, alors que c'est plutôt la réalité extérieure et objective qui est cruciale dans la critique de Thảo.

\section{L'homologie entre linguistique et économie}

Thảo ne se contente pas de critiquer la manière dont Saussure considère la nature des signes qui forment la langue, il lui reproche également d'occulter le processus de constitution de ces signes au profit de l'idée selon laquelle la valeur du signe ne lui viendrait que de la place qu'il occupe dans un système déjà constitué comme un tout. Il propose alors une critique de la linguistique saussurienne sur le modèle de la critique marxienne de l'économie vulgaire ${ }^{4}$, en tant qu'elle s'en tient à la sphère de l'échange pour déterminer la valeur des marchandises, sans prendre la peine de s'intéresser à la sphère de la production. Dans les années 1960-1970, Henri Lefebvre, Ferruccio RossiLandi et Jean-Joseph Goux - on pourrait également ajouter Pierre Bourdieu et JeanPierre Faye à cette liste - ont cherché à élaborer une comparaison semblable entre le champ économique et le champ symbolique fondée sur l'idée d'une homologie, c'est-àdire d'une communauté de structure entre ces deux champs (D'Urso 2014 ; voir aussi l'article du même auteur dans ce volume).

Henri Lefebvre, sociologue et philosophe, déplore, dans Langage et société, l'hégémonie progressive des signes conventionnels sur les symboles, aussi bien dans la théorie que dans la réalité. Les symboles (ou "signes naturels " pour Thảo) sont des signes qui signifient indépendamment de leurs rapports avec les autres signes: ils sont dotés d'une puissance propre et sont chargés d'affects, d'images et de connotations, par contraste avec les signes qui constituent le système saussurien de la langue comme sphère autonome, autoréférentielle et neutre. Or ce qu'Henri Lefebvre (1966: 365) diagnostique comme une «crise du langage » consiste précisément dans le fait que les usages de la langue tendent à lui faire perdre sa dimension symbolique pour la réduire à la structure abstraite que s'efforcent de décrire les linguistes structuralistes: les mots, en ne référant plus que les uns aux autres dans les discours, tendent à tourner à vide et finissent par tomber « dans la trivialité du quotidien ». 
Le discours, dépouillé de symboles, [...] s'établit comme norme sociale. [...] Il se réduit à des implications en apparence logiques, à des imbrications en apparence cohérentes, à des combinaisons laissant place à une sorte de liberté d'indifférence. [...] Chaque élément renvoie à un autre élément, chaque signe à un autre signe, sans commencement ni fin, parce que le discours tourne dans un cercle.

Par la comparaison qu'il propose de faire entre « la forme-marchandise et le discours ", il invite alors à penser la réduction de la valeur des signes à leurs rapports mutuels comme un symptôme semblable à celui qui consiste à réduire une marchandise à sa valeur d'échange, accordant par là une importance secondaire à sa valeur d'usage et occultant le travail qui en a permis la production. C'est l'oubli des origines de la langue qui contribuerait ainsi à la transformer en un système autoréférentiel.

Sans partager son diagnostic, les développements de Ferruccio Rossi-Landi et de JeanJoseph Goux peuvent être considérés comme des continuations de l'intuition de Lefebvre. Dans un article de 1973 (Rossi-Landi \& Murovec 1973) que Thảo a lu (D’Alonzo 2017), Ferruccio Rossi-Landi, sémioticien et philosophe italien, estime que tout signe est un produit (puisqu'il n'existe pas tel quel dans la nature) et qu'à ce titre, il est nécessairement le résultat d'un travail social. Si l'échange linguistique ordinaire tend à masquer le travail dont sont issus les mots que nous utilisons, les cas où la communication rate, eux, le dévoilent. En effet, en faisant l'expérience d'un mot qui ne rend pas bien une idée, on se réfère implicitement à quelque chose qui a été mal fait dans le travail de production de ce mot, de même que « des couteaux qui ne coupent pas, du fil qui se casse à tout moment éveillent chez celui qui les emploie dans un nouveau procès de travail le souvenir d'un autre travailleur, c'est-à-dire de celui qui a fait le couteau et le fil » (Rossi-Landi \& Murovec 1973 : 82).

De la même manière, Jean-Joseph Goux, philosophe français proche du groupe Tel Quel, considère dans Économie et symbolique (1973), qu'il est nécessaire de dépasser l'approche synchronique de la langue pour s'intéresser davantage au "processus de sa stabilisation». L'étude de ce processus ferait apparaitre le caractère essentiel du rapport entre les signes et la réalité, qui avait été occulté par l'étude du système. C'est de ce point de vue que Goux retrace l'histoire de l'écriture: elle témoignerait du passage de signes imitatifs (dessins représentant des réalités singulières) à des hiéroglyphes généraux (dessins qui valent pour toute une catégorie d'objets) puis à l'écriture alphabétique conventionnelle, montrant ainsi que le rapport signe/réalité est à l'origine de la langue telle que nous la connaissons aujourd'hui, origine sans laquelle son fonctionnement actuel resterait incompréhensible.

Comme Goux ou Rossi-Landi, Thảo reproche à Saussure de focaliser son analyse sur les rapports entre des signes déjà constitués plutôt que sur la constitution de ces signes. Cela se manifesterait clairement à la lecture de la comparaison que Saussure propose lui-même d'établir entre les signes linguistiques et la monnaie dont la caractéristique commune serait la valeur. La valeur d'une chose, dit Saussure, est déterminée par des choses dissemblables contre lesquelles elle est susceptible d'être échangée (5 francs $=1$ baguette) et par des choses semblables avec lesquelles on peut la comparer ( 5 francs $=5$ pièces de 1 franc). Ainsi, la valeur d'un mot serait déterminée par l'idée qu'il signifie (qui est d'une nature différente du mot) - c'est sa signification - et par les autres mots de la langue (qui sont de même nature) desquels il se distingue et qui le déterminent négativement - c'est sa valeur au sens strict. 
Thảo critique cette comparaison à la fois du point de vue de son inexactitude (elle repose sur une illusion économique) et du point de vue de ses effets cognitifs aveuglants. Cette comparaison serait inexacte dans la mesure où la monnaie n'est pas d'une nature différente des autres marchandises (Marx 2014 [1867] : 80). Il n'y a pas de différence véritable entre la signification d'un signe et sa valeur, de sorte que vouloir expliquer l'une par l'autre nous enferme dans un cercle, celui de la circulation ou des échanges. En réalité, on présuppose toujours la signification d'un terme lorsqu'on cherche à le distinguer d'autres termes, de même qu'on présuppose toujours une valeur de référence lorsqu'on compare la valeur d'une marchandise $A$ à celle d'une marchandise B. Les rapports d'opposition et de différence entre les signes du système seraient donc déjà des rapports de signification. Thảo adresse ainsi à Saussure le reproche que faisait Marx (2014 [1867]: 92) aux économistes vulgaires qui présupposent «d'abord la valeur d'une marchandise (ici le travail) pour déterminer ensuite par ce moyen la valeur des autres marchandises ", nous empêchant par là l'accès à «l'antre secret de la production" (ibid. : 197). Il faudrait donc sortir du système fermé des signes pour remonter à la détermination de la valeur par la production de ces signes (de même qu'il faut remonter au travail pour déterminer la valeur d'une marchandise) : «il s'agirait, en d'autres termes, d'expliquer le mouvement superficiel des échanges de signes par le mouvement profond de leur production sociale. » (Thảo 1974 : 42).

Ici encore, Thảo cherche donc à identifier l'élément spécifique sur lequel reposent tous les reproches qui ont été adressés à Saussure et à son refus d'affronter la question de l'origine des signes linguistiques. C'est la conception fétichiste de la monnaie et du signe qui serait à l'origine de la théorie saussurienne. Celui-ci, en concevant la valeur marchande comme étant exclusivement déterminée par les échanges de marchandises et la monnaie comme signe conventionnel de la valeur (Molino 1969), en viendrait à considérer la signification des mots du seul point de vue de leurs rapports avec les autres mots et à caractériser les signes comme des conventions. Il semble être en cela influencé par l'école économique de Lausanne. Léon Walras et Ferdinand de Saussure auraient notamment en commun une définition de la valeur (économique dans le premier cas, linguistique dans le second) relativiste plutôt que substantialiste. Pour Walras en effet, qui a donné une forme systématique à la loi de l'offre et de la demande, la valeur d'un bien dépend non seulement de l'état de l'offre et de la demande sur le marché de ce bien mais aussi de l'état de l'offre et de la demande sur les marchés de tous les autres biens. La linguistique saussurienne occulterait donc le travail linguistique créateur de valeur et contribuerait à transformer les signes linguistiques en «hiéroglyphes» indéchiffrables. C'est de là que viendrait prioritairement la nécessité d'en établir la critique. On pourrait ainsi appliquer à la linguistique cette remarque de Marx (2014 [1867] : 104) :

[...] lorsqu'on tient pour de simples signes les caractères sociaux que prennent les choses ou le caractère de choses matérielles que prennent les déterminations sociales du travail sur la base d'un mode de production déterminé, on déclare en même temps qu'on les tient pour des productions arbitraires de la réflexion des hommes ${ }^{5}$. 


\section{Quelle alternative à la linguistique saussurienne ?}

21 Thảo ne se contente pas de critiquer le modèle saussurien. Les deux objections qu'il adresse à Saussure le conduisent à envisager une autre perspective sur la langue, historique plutôt que structurale, et qui seule permettrait de comprendre comment les signes en viennent à recevoir un sens ${ }^{6}$. Plus précisément, cette perspective historique consiste en l'élaboration d'une genèse matérialiste et dialectique du langage et corrélativement de l'intentionnalité et de la conscience, à laquelle Thảo donne le nom de "sémiologie dialectique ». Par opposition à la sémiologie saussurienne, elle repose sur l'hypothèse que la nature des signes ne peut être comprise indépendamment de la manière dont ils ont été produits. Cependant, si on conçoit bien en quoi consiste le travail producteur de marchandises, la production des signes linguistiques, du fait de leur dimension symbolique, est plus difficile à envisager. C'est pourtant bien l'objectif que se fixe Thảo dans ses Recherches sur l'origine du langage et de la conscience.

Si Jean-Joseph Goux limitait son analyse de la genèse du langage à celle de l'écriture (dont la dimension matérielle apparaît de façon plus manifeste et dont l'évolution d'un stade concret à un stade abstrait est à la fois plus récente et plus évidente), Thảo cherche à l'étendre à la communication orale. Cette genèse permettrait alors de ressaisir le processus de production des signes linguistiques et de montrer que cette production ne peut se penser à partir de l'hypothèse de l'arbitraire du signe. En effet, pour penser la constitution de signes linguistiques réduits à des conventions, on est forcé d'envisager cette constitution sous la forme d'un contrat. Si Saussure rejette explicitement la question de l'origine du langage de ses investigations (Saussure 1987 [1916] : 24, 105), il reconnaît cependant que nous pouvons «concevoir» «l'acte par lequel, à un moment donné, les noms seraient distribués aux choses, par lequel un contrat serait passé entre les concepts et les images acoustiques $»^{7}$ et il ajoute que "l'idée que les choses auraient pu se passer ainsi nous est suggérée par notre sentiment très vif de l'arbitraire du signe » (Saussure 1987 [1916] : 105). Or, rappelle Thảo, pour passer un contrat afin de s'accorder sur des conventions linguistiques, il faut déjà être en mesure de parler8. La théorie saussurienne nous enfermerait donc dans un cercle dès lors qu'on chercherait à se baser sur elle pour penser l'origine du langage.

Thảo se propose alors de penser la genèse du langage d'un point de vue "matériel ", autrement que par la fiction d'un contrat basé sur un accord des esprits en faveur du choix arbitraire de tel ou tel signe. La production des signes ne peut se comprendre qu'à la condition qu'on suppose ces signes comme étant, du moins à l'origine, " intrinsèques ", c'est-à-dire comme déterminant "par leur propre structure matérielle les significations fondamentales" (Thảo 1974: 42). Il faut donc supposer que les caractéristiques matérielles des signifiants ont un lien spécifique ("spontané ») avec ce qu'ils sont censés signifier, à savoir la réalité matérielle telle qu'elle se présente à nous lorsque nous y sommes confrontés dans la pratique (ibid.). En effet, la constitution de signes ne peut se penser que par des actes de parole effectifs (qui constitueraient à proprement parler le travail linguistique ${ }^{9}$ ), à l'intérieur desquels les signes utilisés ont un rapport "naturel» avec les réalités qu'ils désignent, rapport sans lequel ils ne pourraient être compris des autres. C'est précisément cette structure matérielle des signes que Thảo propose d'imaginer dans ses Recherches lorsqu'il fait du geste d'indication le cœur de la scène inaugurale qui voit l'émergence de la conscience et du langage. D'un côté, ce geste est le signe le plus élémentaire qu'on puisse imaginer (il 
établit un rapport visuel et physique entre le sujet et l'objet désigné), mais d'un autre côté, il suppose qu'on soit capable de se rapporter à l'objet comme à quelque chose d'extérieur (et non pas seulement comme à quelque chose qu'on peut toucher, voir, etc.), comme à quelque chose donc qui existe indépendant de nous et de nos sensations (Thảo 1973 : 15). C'est ainsi que la genèse du signe linguistique serait en même temps la genèse de la référentialité, de l'intentionnalité et donc de la conscience.

Le geste de l'indication produit donc « entièrement par lui-même, par sa simple forme matérielle, sa propre signification» (ibid.: 39). On pourrait ici reprocher à Thảo de promouvoir l'idéal d'une langue immédiatement référentielle, qui n'introduirait aucune médiation entre la conscience et la réalité. Une des conséquences de cette idée serait que le modèle de la langue ainsi définie ne permettrait pas de rendre compte de notre capacité à mentir ou à parler de ce qui n'existe pas. S'il y a sans doute une part de vérité dans ce reproche, la réponse de Thảo à ces dernières objections serait sans doute que le mensonge n'est possible que parce que nous tenons pour acquis que nos paroles se réfèrent à un monde partagé et qu'on ne peut parler de ce qui n'existe pas qu'avec des mots qui réfèrent à des choses existantes.

Selon Thảo, son hypothèse sur l'origine des signes linguistiques serait la seule à permettre de penser l'interdépendance entre conscience et langue. À l'inverse, supposer des signes d'abord arbitraires impliquerait forcément d'imaginer qu'ils ne sont que l'expression extérieure de significations qui préexistent dans la conscience. C'est pourquoi il faut comprendre le «mouvement sémiotique matériel » comme un mouvement par lequel l'acte signifiant gestuel produit son signifié « en le projetant sur l'objet réel ». C'est seulement sur la base d'un tel mouvement que les significations peuvent ensuite s'autonomiser et être associées à n'importe quel signe verbal, qu'il soit ou non conventionnel.

Comprendre la genèse conjointe du langage et de la conscience revient alors à appliquer ce que Thảo appelle la "méthode objective » ou " dialectique matérialiste " par opposition à la méthode phénoménologique d'un Husserl qui analyse l'expérience vécue de la conscience par la seule "réflexion intérieure de la conscience sur ellemême " (Thảo $1975: 24$ ), plutôt que de partir « des rapports matériels entre l'homme et le monde dans la pratique sociale » (ibid. : 25). Husserl serait en effet conduit par l'usage de cette méthode à réduire la réalité objective à son appréhension subjective, c'est-àdire à la manière dont elle est visée intentionnellement par le sujet. Thảo adresse le même type de reproche à la sémiologie saussurienne, qui, en tant qu'elle réduit le signe à une entité purement psychique réunissant "non une chose [matérielle] et un nom [un son physique], mais un concept et une image acoustique [une représentation idéelle du son physique]", reste enfermée sur le "pur plan idéal de la conscience » et la "sépare complètement de l'activité matérielle des hommes " (ibid.). Ce que montre, à l'inverse, l'analyse du geste de l'indication, c'est la matérialité du «mouvement sémiotique ", par laquelle peut advenir "le moment qui reflète l'objet comme extérieur, l'image du rapport d'extériorité de l'objet par rapport au sujet, l'image de l'extériorité de l'objet » (ibid.) et donc le rapport du sujet à la réalité objective (et non pas seulement aux intentionnalités de la conscience).

Il ne faut donc pas comprendre l'activité productrice de signes comme une activité radicalement différente de toute autre activité productive.

[La] signification consiste à exprimer immédiatement le mouvement même des rapports matériels, et c'est cette signification objective, non consciente que les sujets 
se communiquent tout d'abord entre eux dans leur "comportement matériel ». Cette signification devient subjective quand le sujet se l'exprime à lui-même, dans le langage intérieur ou la conscience. (1973 : 35)

linguistiques: en insistant sur le fait que les signes ont été constitués par les individus et émergent de leurs activités pratiques, on montre en même temps qu'ils peuvent être modifiés par eux. À Saussure qui affirme que «la langue est de toutes les institutions sociales celle qui offre le moins de prise aux initiatives ", on rétorquerait ainsi avec Marx que «les circonstances font tout autant les hommes que les hommes font les circonstances» (Marx \& Engels 1968: 70). À la conception qu'il voit comme éminemment saussurienne de l'inertie et du "poids de la collectivité » (Saussure 1987 [1916] : 108), Thảo oppose donc l'idée marxienne que les individus font leurs langues en parlant, de même qu'ils font leur histoire au moins autant qu'ils sont portés par elle.

\section{Conclusion}

La reconnaissance conjointe de la part active des individus dans la constitution de la langue et de leur capacité à la modifier prend toute son ampleur dès lors qu'on reconnait la centralité des activités langagières dans le monde social. Dans la mesure où l'on admet que la conscience et la pensée n'existent que par le langage, celui-ci, en plus de constituer une activité sociale à part entière, traverse aussi l'ensemble des autres pratiques, pour peu qu'elles soient conscientes. C'est à partir de ce constat qu'on peut souligner l'enjeu principal qui préside à la critique de la linguistique saussurienne telle qu'elle est généralement comprise et telle qu'elle est reçue par Thảo. La linguistique, en effet, en tant que théorie du langage, influence nécessairement, directement ou indirectement, de façon consciente ou non, la manière dont nous nous rapportons à notre propre activité langagière, et en retour cette activité langagière elle-même. Or, en considérant le langage du point de vue de la langue conçue comme système autonome de signes conventionnels, on interdit l'exercice d'un regard critique sur les discours, sur les mots ou sur les expressions que nous entendons et que nous employons. En plaçant hors de toute discussion la possibilité de choisir un signe plutôt qu'un autre ${ }^{10}$, en écartant toute considération sur les rapports entre langage et réalité ou entre langage et société, on met hors de leur champ d'investigation les analyses qui chercheraient à déceler à la fois les illusions dont les discours peuvent être porteurs et les intérêts socio-économiques qui peuvent s'y exprimer. C'est à de telles analyses que se livre par exemple Marx, lorsqu'il critique les discours idéologiques des idéalistes allemands ainsi que certains discours juridiques et économiques. Il explique par exemple comment l'usage de concepts abstraits comme celui d' "homme» ou d' « humanité » peut conduire à masquer les différences sociales et historiques entre les individus réels (Marx \& Engels 1968: 54), ou comment l'utilisation du concept de "propriété » en tant que synonyme de l'idée de possession en général empêche la possibilité même de critiquer la propriété privée, comme phénomène historiquement déterminé (ibid. : 261). À l'inverse, l'insistance sur le caractère de « produits » des mots 
et des significations peut ouvrir la possibilité de réfléchir aux divers usages linguistiques, que ce soit pour y déceler la présence de présupposés idéologiques ou pour les transformer ${ }^{11}$.

\section{BIBLIOGRAPHIE}

Ageeva, Inna. 2009. La critique de F. de Saussure dans Marxisme et philosophie du langage de V.N. Vološinov et le contexte de la réception des idées saussuriennes dans les années 1920-1930 en Russie. Cahiers de l'ILSL 26 : 73-84.

D’Alonzo, Jacopo. 2017. L'origine del linguaggio e della coscienza. Storia di un libro mai pubblicato : dal carteggio inedito tra Ferruccio Rossi-Landi e Trần- Đức- Thảo. Acta Structuralica International Journal for Structuralist Research 2.1 : 87-151.

D’Alonzo, Jacopo. 2018. Trần- Đức- Thảo and the Language of Real Life. Language Sciences 70 : 45-57.

D’Urso, Andrea. 2014. Denaro linguistico e plusvalore ideologico. Krypton 4 : 52-61.

Goux, Jean-Joseph. 1973. Économie et symbolique. Freud, Marx. Paris : Seuil.

Jakubinskij, Lev. 2012. F. de Saussure sur l'impossibilité d'une politique linguistique. Lev Jakubinskij, une linguistique de la parole (URSS, années 1920-1930). Textes édités et présentés par Irina Ivanova, trad. par Irina Ivanova \& Patrick Sériot, 181-212. Limoges \& Lausanne : Lambert-Lucas \& Université de Lausanne.

Lefebvre, Henri. 1966. Langage et société. Paris : Gallimard.

Lénine, Vladimir Ilitch. 1948 [1909]. Matérialisme et empiriocriticisme. Notes critiques sur une philosophie réactionnaire. Paris : Éditions sociales (publication originale : МАТЕРИАЛИЗМ И ЭМПИРИОКРИТИЦИЗМ. КРИТИЧЕСКИЕ ЗАМЕТКИ ОБ ОДНОЙ РЕАКЦИОННОЙ ФИЛОСОФИИ. Moscou : ЗВЕНО. 1909).

Marcellesi, Jean-Baptiste \& Bernard Gardin. 1973. Introduction à la sociolinguistique. Paris : Librairie Larousse.

Marx, Karl. 2011 [1857-1858]. Manuscrits de 1857-1858 dits « Grundrisse ». Paris : Éditions sociales. Marx, Karl. 2014 [1867]. Le Capital (Livre I). Paris : Puf (publication originale : Das Kapital. Kritik der politischen Ökonomie. Hambourg : Otto Meissner. 1867).

Marx, Karl. \& Friedrich Engels. 1968. L'Idéologie allemande. Critique de la philosophie allemande la plus récente dans la personne de ses représentants Feuerbach, B. Bauer et Stirner, et du socialisme allemand dans celle de ses différents prophètes. Paris : Éditions sociales.

Molino, Jean. 1969. Linguistique et économie politique. Sur un modèle épistémologique du cours de Saussure. L'Âge de la science 4 : 335-349.

Rousseau, Jean-Jacques. 1965 [1755]. Discours sur l'origine et les fondements de l'inégalité parmi les hommes , présentation par Bertrand de Jouvenel. Paris : Gallimard. 
Rossi-Landi, Ferruccio \& Ivan Murovec. 1973. Le langage comme travail et comme marché. L'Homme et la société 28 : 71-92.

Saussure, Ferdinand de. 1987 [1916]. Cours de linguistique générale. Éd. par Charles Bally \& Albert Sechehaye, avec la collaboration d'Albert Riedlinger. Paris : Payot (publication originale : Cours de linguistique générale. Lausanne \& Paris : Payot. 1916). N ${ }^{l l e}$ éd. établie par Tullio De Mauro.

Sériot, Patrick. 2020. Lénine, Saussure et la théorie des hiéroglyphes. History and Philosophy of the Language Sciences [https://hiphilangsci.net/2020/05/18/lenine-saussure-hieroglyphes, consulté le $9 / 10 / 2020]$.

Thảo, Trần Đức. 1973. Recherches sur l'origine de la conscience et du langage. Paris : Éditions sociales.

Thảo, Trần Đức 1974. De la phénoménologie à la dialectique matérialiste de la conscience (I). La Nouvelle Critique $79 / 80: 37-42$.

Thảo, Trần Đức 1975. De la phénoménologie à la dialectique matérialiste de la conscience (II). La Nouvelle Critique 86 : 23-29.

Tylkowski, Inna. 2012. Vološinov en contexte. Essai d'épistémologie historique. Limoges : LambertLucas.

Vološinov, Valentin Nicolaievitch. 2010. Marxisme et philosophie du langage. Les problèmes fondamentaux de la méthode sociologique dans les sciences du langage. Éd. et trad. par Inna Tylkowski \& Patrick Sériot. Limoges : Lambert-Lucas (publication originale : МАРКСИЗм И ФИЛосоФИЯ ЯЗЫКА. ОСНОВНЫЕ ПРОБЛЕМЫ СОЦИОЛОГИЧЕСКОГО МЕТОДА В НАУКЕ О ЯЗЫКЕ. Leningrad : Priboj. 1929-1930).

\section{NOTES}

1. Sur les réceptions négatives de Saussure en URSS, voir notamment Sériot 2020.

2. Les individus ne se rapportent pas au mot «cheval » par l'intermédiaire des rapports qu'il entretient avec les autres mots, mais relativement aux circonstances dans lesquelles il est prononcé et qui en déterminent la signification. Pour le locuteur, «le centre de gravité de la langue [se situe] dans le sens concret et toujours nouveau qu'elle prend dans un contexte donné » (Vološinov 2010 : 257).

3. Notons que ces accusations ne tiennent pas compte de la distinction qu'on pourrait établir entre le sens d'un mot à l'intérieur d'un énoncé particulier, déterminé par le contexte et les circonstances d'énonciation, et la signification d'un mot hors contexte, telle qu'on la trouve par exemple dans les dictionnaires.

4. À la différence de l'économie politique classique qui cherche à expliquer le prix des marchandises à partir de la production de leur valeur par le travail, l'économie vulgaire (dont Jean-Baptiste Say, par exemple, est un représentant) se contente, d'après Marx, de systématiser la manière dont les phénomènes apparaissent aux agents économiques.

5. Notons que Marx lui-même refuse la comparaison entre langage et monnaie : voir Marx 2011 [1857-1858]: 121.

6. Bien qu'une perspective historique ne soit pas incompatible avec une approche structuraliste, Thảo considère que la première serait plus essentielle, voire explicative de la seconde.

7. D'un autre côté, Saussure (1987 [1916] : 104) affirme que la langue ne peut « être assimilée à un contrat pur et simple».

8. Il s'agit là d'un argument classique, qu'on trouve notamment chez Rousseau (1965 [1755]: 148-149)

9. C'est aussi l'idée que défend Rossi-Landi (voir D'Urso, ce volume). 
10. «L'arbitraire même du signe met la langue à l'abri de toute tentative visant à la modifier. La masse, fût-elle même plus consciente qu'elle ne l'est, ne saurait la discuter. Car pour qu'une chose soit mise en question, il faut qu'elle repose sur une norme raisonnable. [...] mais pour la langue, système de signes arbitraires, cette base fait défaut, et avec elle se dérobe tout terrain solide de discussion » (Saussure $1987: 106-107)$.

11.

\section{RÉSUMÉS}

Si ses réflexions sur l'origine du langage semblent à première vue relativement éloignées de la problématique saussurienne, Trần Đức Thảo n'en prend pas moins la peine d'en élaborer une critique, en particulier dans deux articles parus dans La Nouvelle Critique en 1974 et 1975. C'est la spécificité de cette lecture et des enjeux qu'elle recouvre que nous nous proposons d'analyser ici. Pour ce faire, il nous semble nécessaire de l'appréhender à l'intérieur du cadre théorique principal dans lequel elle s'inscrit, à savoir la tradition marxiste. Nous verrons ainsi comment la critique de l'abstraction de la théorie saussurienne, d'une part, et la comparaison entre linguistique et économie politique, d'autre part, qu'on retrouve chez un certain nombre d'auteurs marxistes, constituent pour Thảo le pendant négatif d'une conception matérialiste du langage.

At first glance, Trần Đức Thảo's investigations into language origins seem to deeply and essentially differ from Saussure's scientific interests. Nevertheless, Thảo targeted Saussurean semiology, especially in two articles published in La Nouvelle Critique in 1974 and 1975. The following work will map out the main lines of Thả's reading of Saussure. The scope of the present study compels us to take into account the Marxist tradition that constituted the most significant theoretical framework of Thảo's criticism toward Saussure. Given this scenario, we will show how both Thảo's critique of the abstraction of Saussurean theory and the comparison between linguistics and political economy he suggested, along with other Marxist authors, are the negative counterpart of a materialist understanding of language.

\section{INDEX}

Mots-clés : Thảo (Trần Đức), Saussure (Ferdinand de), langage, marxisme, linguistique

Keywords : Thảo (Trần Đức), Saussure (Ferdinand de), language, marxism, linguistics

\section{AUTEUR}

\section{JULIETTE FARJAT}

Université Paris Nanterre, Laboratoire de sociologie, philosophie et anthropologie politiques (EA 3932, Sophiapol), Nanterre, France 